\title{
A Comparative Study of Health Anxiety in Neurology and Psychiatry Settings
}

\author{
Lakshmi Prabha ${ }^{1}$ Sundarnag Ganjekar ${ }^{2}$ Vivek Gupta ${ }^{3}$ Geetha Desai ${ }^{2}$ Santosh K. Chaturvedi
}

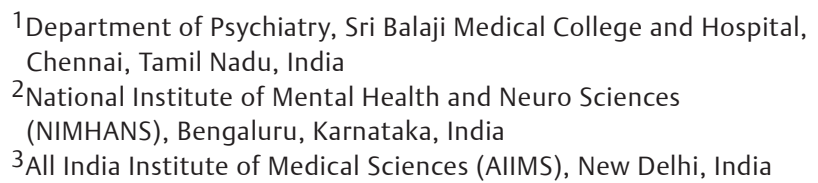

Address for correspondence Geetha Desai, MD, PhD, National Institute of Mental Health and Neuro Sciences (NIMHANS), Bengaluru 560029, Karnataka, India (e-mail: desaigeetha@gmail.com).

J Neurosci Rural Pract 2020;11:125-129

\begin{abstract}
Objectives Prevalence of health anxiety is highly varied based on different settings and samples studied. This study aimed to assess the prevalence of health anxiety among outpatients attending neurology and psychiatry outpatient setting in tertiary care hospital and understand the clinical correlates.

Participants and Methods This was a cross-sectional study conducted in the outpatient settings including participants fulfilling study criteria. The assessments included semi-structured proforma for demographic and clinical details, general hypochondriasis subscale of Illness Behavior Questionnaire, and Short Health Anxiety Inventory. Data were collected in Epi-info and data analysis was done using STATA12.

Results The health anxiety was reported to be $25 \%$ and $19 \%$ among psychiatry and neurology outpatients, respectively. Higher education level positively correlated with health anxiety. Skilled workers tend to have higher health anxiety than semiskilled

Keywords

- health anxiety

- psychiatry

- neurology

- outpatient workers. Patients with diagnosis of somatoform disorder and multiple diagnosis scored higher on health anxiety in both the settings.

Conclusion Health anxiety appears to be common in psychiatry and neurology settings and needs further evaluation to understand its impact on consultation and health resource usages.
\end{abstract}

\section{Introduction}

Heath anxiety has been described as "concern about health in the absence of pathology or excessive concern when there is some degree of pathology."1 It is associated with functional impairment, as well as inappropriate utilization of health care services. The severe form of health anxiety constitutes hypochondriasis. ${ }^{2}$

Significant variation in the prevalence rates of health anxiety within different medical settings has been reported. A study conducted to assess the prevalence of pathological health anxiety across different specialities of medical outpatient clinics using Short Health Anxiety Inventory (SHAI) reported that around $20 \%$ were found to have significant health anxiety. ${ }^{3}$ A prevalence of $36.7 \%$ for hypochondriasis and $51.1 \%$ for severe and disabling health anxiety was reported in chronic pain patients in a comparative study of health anxiety among

DOI https://doi.org/

$10.1055 / \mathrm{s}-0039-3399395$

ISSN 0976-3147. patients attending chronic pain clinic with controls using SHAI. ${ }^{4}$ Studies on health anxiety in diabetes mellitus and multiple sclerosis have reported high levels of health anxiety. ${ }^{5,6} \mathrm{~A}$ community study done in German population $(n=2198)$ reported that only $0.4 \%$ fulfilled the Diagnostic and Statistical Manual of Mental Disorders, 4th Edition, criteria for hypochondriasis and approximately $6 \%$ of subjects found to have high health anxiety. ${ }^{7}$ Another epidemiological study in Australian population among 8841 respondents reported that $3.4 \%$ of the sample had health anxiety at the time of assessment and $5.7 \%$ had health anxiety at some point in their life. ${ }^{8}$ In a review done on epidemiology of health anxiety and hypochondriasis by using different diagnostic criteria, health anxiety reported in the general population had a wide range (2.1-13.1\%). Weighted prevalence of hypochondriasis was around $0.40 \%$ (range: $0.0-4.5 \%$ ) and abridged hypochondriasis $1.00 \%(0.6-2.0 \%)$ in general population. ${ }^{9}$ These variations in the prevalence of health anxiety is due to different 
specialties, settings, and different medical diagnosis. There is minimal research in the area of health anxiety in developing countries such as India. This study aimed to assess prevalence and clinical correlates of health anxiety among patients attending neurology and psychiatry outpatient clinics.

\section{Methods}

The objectives of the study included assessment of the health anxiety in patients attending neurology and psychiatry settings and studying the demographic and clinical correlates of health anxiety. It was a cross-sectional study with sample being chosen from patients attending outpatients' services of psychiatry and neurology at NIMHANS, Bengaluru, India. The inclusion criteria for the study were gender, age more than 18 years, and patients who could understand Kannada, Tamil, or English. Patients with a diagnosis of severe mental illness were excluded. Patients were excluded if they could not complete the survey because of mental or physical incapacity. Based on reported prevalence of health anxiety as $20 \%$, a relative error of $20 \%$, and $5 \%$ type- 1 error probability, a target sample size of 400 patients was required in each setting. Eligible patients were identified by systematic random sampling from psychiatry and neurology outpatient services.

After obtaining written informed consent, the patients were interviewed and information on demographics (age, sex, education level, socioeconomic status, occupation), clinical symptoms, comorbid conditions, and diagnoses was obtained. This was followed by administration of, self-report questionnaire of SHAI, and general hypochondriasis subscale of Illness Behaviour Questionnaire.

\section{Short Health Anxiety Inventory}

It is a self-report 18-item questionnaire that assesses health concerns over a period of 6 months that includes domains of illness worry, disease conviction, and negative consequences, rated on a Likert scale from 0 to 3 . The SHAI has demonstrated good reliability, criterion validity, and sensitivity to treatment. ${ }^{10}$ The SHAI was translated and back translated following standard procedures of translation.

\section{Illness Behavior Questionnaire}

It is a self-administered questionnaire that uses a "yes"|"no" response format. The expanded version contains 62 items of which 11 of the questions were taken from the general hypochondriasis subscale of Illness Behaviour Questionnaire. It assesses the maladaptive responses to illness including hypochondriacal responses. ${ }^{11,12}$

\section{Data Analysis}

Data entry was done using Epi-info $7.0^{13}$ and data analysis using Stata $12 .{ }^{14}$ Descriptive analyses were done with mean (standard deviation), median (interquartile range), or proportions (\%) as appropriate. The responses received for each option of Illness Behavior Questionnaire (IBQ) and SHAI scales were tabulated separately for patients from neurology and psychiatry. Test scores on each scale were computed as were the proportion of patients with threshold scores. Only the first 14 items of the SHAI that assess the illness worry and body vigilance were included while estimating the overall score and prevalence, because the last four items assessed the perceived negative consequences of developing a serious illness that may not be applicable for patients already having a serious illness as reported in earlier studies (medical samples). Correlations between IBQ and SHAI were assessed using $r^{2}$ values. Respondents from neurology and psychiatry outpatient department were compared using chi-squared tests for proportions and $t$-tests for scores. The statistical significance level was considered at $p<0.05$.

\section{Results}

A total 843 patients were assessed in the study of which 431 were in psychiatry setting and 412 were in neurology setting. The mean age of participants was $37 \pm 11.77$ years; being lower in the neurology group $(35 \pm 11.6)$ than psychiatry group (39 \pm 10.95$)(t=5.44 ; p<0.01)$. Women composed $49 \%$ (411) of the total sample, with more women in psychiatry group than neurology group $(p<0.05)$. Majority of them were from urban background (527, $62.5 \%$ ), while $13.4 \%$ (113) were illiterate. In the total sample, $11.2 \%$ (95) participants were unemployed, 23.7\% (200) were home-makers, and the rest were skilled and semiskilled workers. Majority of the sample belonged to upper lower $(51 \%, 418)$ and lower middle $(25.4 \% ; 208)$ socioeconomic status ( - Table $\mathbf{1}$ ).

In the total sample, the most common primary diagnosis was seizure disorder (26\%) followed by depression (17\%), anxiety disorder (15\%), somatoform disorder (15\%), in that order. The most common diagnosis in psychiatry sample was depression (32\%) and the most common diagnosis in neurology was seizure disorder (52\%). Patients in psychiatry group had more than one diagnosis (second diagnosis). In neurology, the most common additional diagnosis (second diagnosis) was other psychiatry diagnosis (3\%) followed by seizure disorder $(2 \%)$ and headache disorder $(1 \%)$ in that order, respectively. Sixteen percent of the sample had comorbidities such as cardiovascular disorders, thyroid disorders, diabetes mellitus, tuberculosis, and others apart from their primary psychiatric or neurological diagnosis.

The mean SHAI score in psychiatry and neurology was $14.76 \pm 8.01$ and $13.17 \pm 7.23$, respectively, while the mean SHAI score in the total sample was $13.98 \pm 7.67$. This difference in the mean SHAI scores was statistically significant. With cutoff score of SHAI $\geq 20$, the prevalence of health anxiety in psychiatry was $25 \%$ that was more than the prevalence of health anxiety in neurology (i.e., 19\%), with an overall prevalence of $22 \%$. The difference in the two groups was significant. With a cutoff score of $\geq 27$ for 14 items, the prevalence estimates were $9 \%$ in psychiatry and $5 \%$ in neurology that were again statistically significant.

The mean SHAI score was compared with age, gender, background, marital status, education level of the patient, and socioeconomic status. Only education level correlated with SHAI scores. Higher the education, higher were the scores on 
Table 1 Demographic and clinical characteristics of enrolled patients

\begin{tabular}{|c|c|c|c|c|}
\hline & Psychiatry $(n=431)$ & Neurology $(n=412)$ & Total $(n=843)$ & $p$-Value \\
\hline Age (mean [SD]) & $39(10.5)$ & $35(11.6)$ & $37(11.8)$ & $<0.01$ \\
\hline \multicolumn{5}{|l|}{ Gender } \\
\hline \multicolumn{5}{|l|}{ Male } \\
\hline Female & $240(55.7)$ & $171(41.6)$ & $411(48.8)$ & $<0.05$ \\
\hline \multicolumn{5}{|l|}{ Education } \\
\hline Illiterate & $81(18.7)$ & $32(7.7)$ & $113(13.4)$ & $<0.01$ \\
\hline Primary/middle/high & $221(51.3)$ & $217(52.6)$ & $438(51.9)$ & \\
\hline Intermediate/post-high & $129(29.9)$ & $163(39.5)$ & $292(34.6)$ & \\
\hline \multicolumn{5}{|l|}{ Occupation } \\
\hline Unemployed & $48(11.1)$ & $47(11.4)$ & $95(11.2)$ & $<0.01$ \\
\hline Unskilled & $92(21.3)$ & $26(6.3)$ & 118 (13.9) & \\
\hline Semiskilled & $84(19.5)$ & $128(31.1)$ & $212(25.1)$ & \\
\hline Skilled & $56(12.9)$ & $57(13.8)$ & $113(13.4)$ & \\
\hline Clerical/ business/professional & $46(10.7)$ & $58(14.1)$ & $104(12.3)$ & \\
\hline Housewife & $104(24.1)$ & $96(23.3)$ & $200(23.7)$ & \\
\hline \multicolumn{5}{|l|}{$\begin{array}{l}\text { Socioeconomic status (modified } \\
\text { Kuppuswamy Scale) }\end{array}$} \\
\hline Lower & $3(0.7)$ & $4(1.0)$ & $7(0.9)$ & 0.001 \\
\hline Upper lower & $235(57.7)$ & $183(44.4)$ & $418(51.0)$ & \\
\hline Lower middle & $86(21.1)$ & $122(29.6)$ & $208(25.4)$ & \\
\hline Upper middle & $68(16.7)$ & $87(21.1)$ & 155 (18.9) & \\
\hline Upper & $15(3.7)$ & $16(3.9)$ & $31(3.8)$ & \\
\hline $\begin{array}{l}\text { Monthly family income (median } \\
{[\mathrm{IQR}] \text { ) }}\end{array}$ & $\begin{array}{l}7000 \\
(5000-15000)\end{array}$ & $\begin{array}{l}10000 \\
(5000-16000)\end{array}$ & $\begin{array}{l}9000 \\
(5000-15000)\end{array}$ & 0.02 \\
\hline \multicolumn{5}{|l|}{ Symptom profile } \\
\hline No symptoms & $96(22.3)$ & $228(55.3)$ & $324(38.4)$ & $<0.01$ \\
\hline Only physical & $273(63.3)$ & $125(30.3)$ & $398(47.2)$ & \\
\hline Only psychological & $35(8.1)$ & $53(12.9)$ & $88(10.4)$ & \\
\hline $\begin{array}{l}\text { Both physical and } \\
\text { psychological }\end{array}$ & $27(6.3)$ & $6(1.5)$ & $33(3.9)$ & \\
\hline \multicolumn{5}{|l|}{ Primary diagnosis } \\
\hline Only neurology & $35(8.1)$ & $393(95.4)$ & $428(50.8)$ & - \\
\hline Neurology and psychiatry & $32(7.4)$ & $16(3.9)$ & $48(5.7)$ & \\
\hline Only psychiatry & $364(84.5)$ & $3(0.7)$ & $367(43.5)$ & \\
\hline \multicolumn{5}{|l|}{ Comorbidities } \\
\hline Cardiovascular & $35(8.1)$ & $28(6.8)$ & $63(7.5)$ & 0.46 \\
\hline Diabetes mellitus & $28(6.5)$ & $12(2.9)$ & $40(4.7)$ & 0.01 \\
\hline Thyroid disorders & $1(0.2)$ & $20(4.9)$ & $21(2.5)$ & $<0.01$ \\
\hline Prior consultations & $253(58.7)$ & $197(47.8)$ & $450(53.4)$ & $<0.01$ \\
\hline
\end{tabular}

Abbreviations: IQR, interquartile range; SD, standard deviation.

SHAI indicating high levels of health anxiety. No other demographic variables correlated with SHAI scores. Skilled workers had higher scores on SHAI than semiskilled workers.
The mean SHAI score of patients with both neurological and psychiatric diagnosis (15.91) was higher than the patients with only neurological or only psychiatric 
Table 2 Sociodemographic correlates of scores on SHAI

\begin{tabular}{|l|l|l|l|l|l|l|}
\hline \multirow{2}{*}{} & \multicolumn{3}{|c|}{ Psychiatry } & \multicolumn{3}{c|}{ Neurology } \\
\cline { 2 - 7 } & $n$ & Score & p-Value & $n$ & Score & $p$-Value \\
\hline & & & & & Mean $(95 \%$ Cl) & \\
\hline Gender & & & & & \\
\hline Male & 240 & $15.4(14.2,16.6)$ & 0.15 & 171 & $12.9(11.8,13.9)$ & 0.52 \\
\hline Female & 191 & $14.3(13.3,15.2)$ & & 240 & $13.3(12.4,14.3)$ & \\
\hline Education & & & & & & \\
\hline Illiterate & 81 & $12.8(11.1,14.5)$ & 0.03 & 32 & $11.4(9.6,13.2)$ & 0.24 \\
\hline Primary-high school & 221 & $14.9(13.8,15.9)$ & & 217 & $13.0(12.0,14.0)$ & \\
\hline Intermediate school or higher & 129 & $15.7(14.3,17.1)$ & & 163 & $13.7(12.6,14.8)$ & \\
\hline Occupation & & & & & & \\
\hline Unemployed & 48 & $14.3(12.0,16.5)$ & 0.01 & 47 & $12.79(10.68,14.89)$ & 0.62 \\
\hline Unskilled & 92 & $12.7(11.3,14.1)$ & & 26 & $12.04(9.52,14.56)$ & \\
\hline Semiskilled & 84 & $17.1(15.3,18.8)$ & & 128 & $13.53(12.22,14.84)$ & \\
\hline Skilled & 56 & $16.3(13.9,18.6)$ & & 57 & $14.42(12.53,16.31)$ & \\
\hline Clerical/business/Prof/farmer & 46 & $14.2(11.8,16.6)$ & & 58 & $13.09(11.38,14.79)$ & \\
\hline Housewife & 104 & $14.2(12.7,15.7)$ & & 96 & $12.53(11.07,13.99)$ & \\
\hline Physical symptoms & & & & & & \\
\hline No & 131 & $13.01(11.5,14.5)$ & 0.01 & 281 & $12.8(11.9,13.6)$ & 0.08 \\
\hline Yes & 300 & $15.5(14.7,16.4)$ & & 131 & $14.1(13.0,12.2)$ & \\
\hline Psychological symptoms & & & & & & \\
\hline No & 369 & $14.4(13.6,15.2)$ & 0.02 & 353 & $12.8(12.0,13.5)$ & 0.01 \\
\hline Yes & 62 & $16.9(14.5,19.3)$ & & 59 & $15.6(13.5,17.6)$ & \\
\hline
\end{tabular}

Abbreviations: $\mathrm{Cl}$, confidence interval; SHAI, Short Health Anxiety Inventory.

diagnosis. Patients with diagnosis of somatoform disorders had higher scores on SHAI compared with other diagnosis.

The SHAI scores had no significant correlation with age of the patients both in psychiatry (correlation $=0.034, p=0.48$ ) and in neurology (correlation $=0.035, p=0.48)$ ( - Table 2).

There was a significant correlation between the 14-item SHAI score and IBQ score in psychiatry (correlation $=+0.63$, $\mathrm{p}<0.01$ ) and in neurology (correlation $=+0.57, \mathrm{p}<0.01$ ), and overall among all respondents (correlation $=+0.61, \mathrm{p}<0.01$ ). On linear regression, each unit increase in IBQ was associated with 1.54 (95\% confidence interval $[\mathrm{CI}]: 1.36,1.72$ ) points increase in SHAI score in psychiatry respondents, while the corresponding change in neurology respondents was 1.32 (95\% CI: $1.14,1.51)$ points and 1.42 (95\% CI: $1.30,1.55)$ points among all respondents.

\section{Discussion}

Our study reported a prevalence rate of health anxiety was $25 \%$ in psychiatry setting and $19 \%$ in neurology settings when a cutoff of 20 was used. There are variations in different studies with regard to cutoff score for assessing health anxiety and also regarding 14 or 18 items as there was a controversy that the last four items that assessed the perceived negative consequences may not be applicable in medical settings in certain studies. Considering these facts, in this study we included only the first 14 items for studying the prevalence in both the settings. The two groups differed significantly on most of the demographic variables, and hence the differences in the prevalence may not be due to the setting alone.

The prevalence of health anxiety in neurology was lower in our study compared with the previous study in neurology setting. ${ }^{3}$ The rates of higher health anxiety in psychiatry group could be due to the fact that majority of the patients had a diagnosis of somatoform disorders who were reported to have high health anxiety. A cutoff score of $\geq 27$ also has been recommended in previous studies especially to discriminate patients with hypochondriasis from other anxiety disorders using a 18 -item version of SHAI. ${ }^{15}$ Utilizing this cutoff in our study and including only the first 14 items revealed a prevalence of approximately $9 \%$ in psychiatry setting and $5 \%$ in neurology setting. Despite the use of higher cutoff score, the psychiatry group had higher health anxiety than neurology group that has been demonstrated in previous study. ${ }^{16}$ 
When the demographic characteristics of the groups were examined, women were more in psychiatry group (56\%) when compared with neurology (42\%) that was significant. However, in the overall sample there were no gender differences. This difference could be due to the fact that the psychiatry group was represented by patients with anxiety, depression, and somatoform disorders whose prevalence has been noted to be high in women than in men. ${ }^{16}$ Majority of the patients were from urban background (63\%) in both the groups. The sample is chosen from a tertiary care center located in an urban setting that predominantly caters to urban population. Higher education was associated with higher health anxiety, possibly due to higher awareness of the illness or consequences of an illness.

Age did not have any correlation with health anxiety score that is in accordance with earlier studies. ${ }^{4}$ Many studies have reported higher rates of health anxiety in women ${ }^{17}$ this study did not find any difference and gender did not have any correlation with health anxiety. Though female patients were more in psychiatry (56\%) than neurology, the mean SHAI score was higher in males than in females.

Patients with a diagnosis of somatoform disorder had a statistically significant high health anxiety scores (17.49) than patients without somatoform disorder (13.38). This is an expected outcome as patients with somatoform disorders are overly concerned about their symptoms. It has been found in our study that patients having more than one diagnosis had high health anxiety scores (15.65) than patients with a single diagnosis (13.80). Patients who had both neurological and psychiatric diagnosis had high health anxiety score (15.91) than patients with only psychiatric diagnosis (14.83) or only neurological diagnosis (13.04). Though health anxiety scores have been compared across different symptoms and diagnosis of the patients, there is difficulty in comparing with other studies as there is paucity of data on the same. There is also a heterogeneity of the scores among various diagnosis in medical settings. Also, in our study only few patients had an already established hypochondriacal disorder diagnosis and they have been clubbed under somatoform disorder diagnosis group. Because of this, the difference in score between patients with hypochondriasis and other anxiety disorder diagnosis could not be compared as done in previous studies. Education, somatoform disorders, and multiple diagnosis significantly influenced the prevalence of health anxiety.

The strengths of the study include it being done on a large sample, using tools that are psychometrically validated. The limitations of the study include that even though the tools are psychometrically sound in measuring the construct of health anxiety, they need to be validated in our setting. It is a cross-sectional study and hence the influence of disease status and duration of illness could not be ascertained. The cutoff score is not known in Indian settings. Though the tools have been reported to have good discriminant validity, considering the overlap of health anxiety in different disorders the presence of underlying disorders also might have influenced the score to some extent, which needs consideration.
Health anxiety appears to be common among patients attending psychiatry and neurology settings and may need further evaluation to understand its impact on consultations and health resources usage.

\section{Funding}

None.

\section{Conflict of Interest}

None declared.

\section{References}

1 Salkovskis PM, Warwick HM. Morbid preoccupations, health anxiety and reassurance: a cognitive-behavioural approach to hypochondriasis. Behav Res Ther 1986;24(5):597-602

2 Abramowitz JS, Olatunji BO, Deacon BJ. Health anxiety, hypochondriasis, and the anxiety disorders. Behav Ther 2007;38(1):86-94

3 Tyrer P, Cooper S, Crawford M, et al. Prevalence of health anxiety problems in medical clinics. J Psychosom Res 2011;71(6):392-394

4 Rode S, Salkovskis P, Dowd H, Hanna M. Health anxiety levels in chronic pain clinic attenders. J Psychosom Res 2006;60(2):155-161

5 Kehler MD, Hadjistavropoulos HD. Is health anxiety a significant problem for individuals with multiple sclerosis? J Behav Med 2009;32(2):150-161

6 Janzen Claude JA, Hadjistavropoulos HD, Friesen L. Exploration of health anxiety among individuals with diabetes: prevalence and implications. J Health Psychol 2014;19(2):312-322

7 Bleichhardt G, Hiller W. Hypochondriasis and health anxiety in the German population. Br J Health Psychol 2007;12(Pt 4) :511-523

8 Sunderland M, Newby JM, Andrews G. Health anxiety in Australia: prevalence, comorbidity, disability and service use. Br J Psychiatry 2013;202(1):56-61

9 Weck F, Richtberg S, Neng J. Epidemiology of hypochondriasis and health anxiety: comparison of different diagnostic criteria. Curr Psychiatry Rev 2014;10(1):14-23

10 Salkovskis PM, Rimes KA, Warwick HM, Clark DM. The Health Anxiety Inventory: development and validation of scales for the measurement of health anxiety and hypochondriasis. Psychol Med 2002;32(5):843-853

11 Varma VK, Malhotra AK, Chaturvedi SK. Illness behaviour questionnaire (IBQ): translation and adaptation in India. Indian J Psychiatry 1986;28(1):41-46

12 Prior KN, Bond MJ. The measurement of abnormal illness behavior: toward a new research agenda for the Illness Behavior Questionnaire. J Psychosom Res 2008;64(3):245-253

13 Epi InfoTM: Centers for Disease Control and Prevention. [Online]. Available at: https://www.cdc.gov/epiinfo/index. html. Accessed December 18, 2018

14 Stata: Software for Statistics and Data Science. [Online]. Available at: https://www.stata.com/. Accessed December 18, 2018

15 Alberts NM, Hadjistavropoulos HD, Jones SL, Sharpe D. The Short Health Anxiety Inventory: a systematic review and meta-analysis. J Anxiety Disord 2013;27(1):68-78

16 Shidhaye R, Mendenhall E, Sumathipala K, Sumathipala A, Patel V. Association of somatoform disorders with anxiety and depression in women in low and middle income countries: a systematic review. Int Rev Psychiatry 2013;25(1):65-76

17 MacSwain KLH, Sherry SB, Stewart SH, et al. Gender differences in health anxiety: an investigation of the interpersonal model of health anxiety. Pers Individ Dif 2009;47(8):938-943 